\section{Prävention des Prostatakarzinoms}

Manfred P. Wirth, Oliver W. Hakenberg

Klinik und Poliklinik für Urologie, Technische Universität Dresden

\section{Prevention of prostate cancer}

Prostate cancer has become the most frequently diagnosed male cancer next to non-melanotic skin cancer in the Western world. Preventive measures would therefore have important potential effects on the incidence and prevalence of this disease. A potential for effective prevention of prostate cancer is currently seen in dietary changes and perhaps in dietary supplementation with vitamins $D$ and $E$ or selenium. Pharmacological prevention seems a possibility with drugs acting on intraprostatic testosterone metabolism. Several large randomised trials are ongoing to clarify the potential for successful prostate cancer prevention.

\section{eingereicht: 01.04.2005 akzeptiert: 30.06.2005}

Das Prostatakarzinom ist das häufigste Organmalignom des Mannes und damit eine Erkrankung, bei der eine wirksame präventive Maßnahme bedeutende Auswirkungen auf Inzidenz, Prävalenz und krankheitsspezifische Mortalität haben könnte. Mehrere Umstände lassen präventive Maßnahmen potentiell erfolgreich erscheinen. Erstens beinhaltet das gegenwärtige Verständnis der molekularen Mehrschrittpathogenese des Prostatakarzinoms mehrere histologische präkanzeröse Veränderungen, welche sowohl genetische als auch epigenetische Veränderungen tragen, und die deutlich früher als das manifeste Prostatakarzinom auftreten. So finden sich prostatische intraepitheliale Neoplasien(PIN) in Autopsiestudien bereits bei Männern unter 30 Jahren, während das manifeste Prostatakarzinom selten unterhalb von 50 Jahren auftritt.

Zum zweiten ergeben sich aus epidemiologischen Untersuchungen bedeutsame Unterschiede in der weltweiten Inzidenz des Prostatakarzinoms. Diese lag 2002 in Deutschland bei 87/100 000 (Krebsregister, A. b. Krebs in Deutschland. Häufigkeit und Trends 2004) und liegt innerhalb Europas in den nördlichen Ländern deutlich höher als in den südlichen (Schweden 55/100 000, Italien 26/100 000) (Grönberg H: Lancet 2003; 361: 859-864). Entsprechende regionale Un- terschiede finden sich für die Mortalität am Prostatakarzinom(33/100 000 in Schweden, 13/100 000 in Spanien (Landis SH, Murray T et al. Cancer statistics 1999: Ca Cancer J Clin 1999; 49: 8-15)). In Asien sind Inzidenz und spezifische Mortalität wesentlich niedriger als in Europa oder den USA (Inzidenz in China 2/100 000, in Japan 10/100 000 (Grönberg H 2003), Prostatakarzinomspezifische Mortalität in Japan 4/100 000 (Landis SH, Murray Tet al. Ca Cancer JClin 1999; 49: 8-15). Die Inzidenz des Prostatakarzinoms steigt jedoch bei asiatischen Einwanderern in die USA in der zweiten Generation deutlich an und liegt für japanische Einwanderer der zweiten Generation bei 47/100 000 (Muir CS, Nectoux J et al. Acta Oncol 1991; 30: 133-139; Grönberg H. Lancet 2003; 361: 859-864). Diese beiden epidemiologischen Beobachtungen werden seit längerem als Hinweis auf die Bedeutsamkeit von Umweltfaktoren, insbesondere der Ernährung für die Entwicklung eines Prostatakarzinoms angesehen.

Drittens beinhaltet die Entwicklung des langsam wachsenden Prostatakarzinoms eine lange präklinische Latenzzeit, so dass ausreichend Zeit für die Wirkung einer effektiven präventiven Maßnahme gegeben erscheint. In mehreren Studien wird daher gerade die Chemoprävention durch Nahrungssupplemente oder Medikamente geprüft.
Tab. 1 Nahrungsinhaltsstoffe mit Bedeutung für die Entstehung eines Prostatakarzinoms.

\begin{tabular}{|ll}
\hline Präventiv wirksam & fördernd \\
\hline Selen & Fleisch \\
\hline Soja & Fette, v.a. gesättigte \\
\hline Vitamine D, E & Fettsäuren \\
\hline Lycopene (Tomaten) & hoher Energiegehalt \\
\hline Fisch & hohe Kalziumzufuhr \\
\hline Gemüse, v.a. Kreuzblüter & Milch \\
\hline Getreideprodukte & \\
\hline Hülsenfrüchte & \\
\hline Fruchtzucker, Obst & \\
\hline
\end{tabular}

Chemoprävention durch Ernährung

Die sehr niedrige Inzidenz des Prostatakarzinoms in China und Japan lenkte in den 1990er Jahren das Interesse auf Unterschiede in der asiatischen und westlichen Ernährung als bedeutsam für die Auslösung eines Prostatakarzinoms. Die asiatische Ernährung ist wesentlich gemüsereicher, fleisch- und fettärmer und dabei ballaststoffreicher als die westliche. Zahlreiche Untersuchungen haben ergeben, dass dabei besondere Bedeutung den Isoflavonen und Lycopenen sowie der Sojamilch und dem grünen Tee zukommt (Klein EA. Nat Clin Prac Urol 2005; 2: 24-31).

\section{Sojamilch}

Isoflavone (Genistein ist das prädominante Isoflavon in Sojamilch) besitzen eine schwach estrogene Wirkung. Darüber hinaus enthält Sojamilch verschiedene andere antikarzinogen wirksame Inhaltsstoffe (Phytosterole, Saponine, Lignane, Proteaseinhibitoren). In mehreren Tiermodellen für das Prostatakarzinom konnte ein proliferationshemmender Effekt von Sojabohnen-basierter Ernährung nachgewiesen werden (Klein EA. Prostate cancer. Clinical preventive medicine 2004. Lang RS and Hansrud DD, AMA Press) und epidemiologische Untersuchungen stützen die Hypothese der präventiven Wirksamkeit der Sojabohne (Severson RK et al. Cancer Res 1989; 49(7): 1857-1860; Adlercreutz H et al. Am J Clin Nutr 1991; 54 : 1093-1100; Adlercreutz H et al. Lancet 1993; 342: 1209-1210; Herbert JR. J Natl Cancer Inst 1998; 90: 1637-1647). Eine große prospektive Untersuchung mit über 12000 Teilnehmern zeigte eine 70\%ige Reduktion des Pro- 
statakarzinomrisikos bei täglicher Einnahme von Sojamilch (Jacobsen BK et al. Cancer Causes Control 1998; 9: 553-557).

\section{Tomaten, rote Früchte, grüner Tee}

Lycopen ist ein ungesättigtes Isomer von $\beta$-Caroten, das primär in Tomaten sowie anderen roten Früchten und Gemüse vorkommt. Es besitzt antioxidative Eigenschaften und ist das im menschlichen Plasma dominierende Carotenoid. Eine Risikoreduktion für das allgemeine Krebsrisiko durch Lycopene scheint aufgrund verschiedener Studien wahrscheinlich zu sein (Giovannucci E, Clinton SK. J Natl Cancer Inst 1999; 91: 317331). Für das Prostatakarzinom wird ebenfalls eine Risikoreduktion von 30-40\% geschätzt, jedoch ist die Evidenz aufgrund gegenteiliger Ergebnisse verschiedener Untersuchungen nicht eindeutig (Giovannucci E, Rimm EB et al. J Natl Cancer Inst 2002; 94: 391-398). Untersuchungen zum grünen Tee haben sich bisher auf die enthaltenen Polyphenole konzentriert. Hierzu liegen bislang lediglich Untersuchungen vor, die einen proliferationshemmenden Effekt von Polyphenolen in Zellkulturen zeigen (Adhami VM et al. J Nutr 2003; 133 Suppl: 241S-242S).

\section{Viel Fisch, wenig Fleisch und Fett}

Ein präventiver Einfluss einer fleisch- und fettarmen sowie fischreichen Ernährung wird aufgrund der epidemiologischen Datenlage ebenfalls vermutet. Hierzu liegen zum Teil widersprüchliche Untersuchungen vor (Terry PD, Rohan TE et al. Am J Clin Nutr 2003; 77:532-543).Jedoch konnten einzelne Untersuchungen eine Verminderung des Progressionsrisikos eines manifesten und fortgeschrittenen Prostatakarzinoms nach einer entsprechenden Ernährungsumstellung zeigen (Fradet $Y$, Meyer F et al. Eur Urol 1999; 35: 388-391). Eine Veränderung der Androgene und der PSA-Parameter konnte bei Patienten mit Prostatakarzinom durch Nahrungssupplementation gezeigt werden (Kranse R, Dagnelie PC et al. Int J Cancer 2005; 113: 835-840). Ein deutlicher Hinweis fand sich in der „Health Care Professionals Follow-up Studie“, in der eine signifikante Risikoreduktion für das Auftreten eines Prostatakarzinoms, insbesondere in metastasierter Form, in Abhängigkeit von der Häufigkeit des wöchentlichen Fischverzehrs und der Aufnahme von marinen Fettsäuren nachgewiesen werden konnte (Augustsson K,
Michaud DS et al. Cancer Epidemiol Biomarkers Prev 2003; 12: 64-67). Ob sich hieraus tatsächlich eine klinisch bedeutsame kotherapeutische Option bei der Therapie eines manifesten Prostatakarzinoms ableiten lässt, ist bislang nicht bewiesen.

\section{Vitamin D, Vitamin E, Selen}

Von Bedeutung bei der Prävention des Prostatakarzinoms erscheinen auch die Vitamine D und E sowie Selen. Der deutliche epidemiologische Inzidenzunterschied zwischen nord- und südeuropäischen Ländern, eine erhöhte Prostatakarzinominzidenz und -mortalität bei Männern mit reduzierten Spiegeln an aktivem Vitamin D und präklinische antiproliferative Effekte von Vitamin D auf Prostatakarzinomzellen sind nachgewiesen (Peehl DM et al. J Nutr 2003; 133: 2461S-2469S). Präventionsstudien liegen hierzu bislang nicht vor, wobei sich neuere Vitamin D-Analoga mit geringer hyperkalzämischer Wirkung in der klinischen Erprobung befinden.

Vitamin E (-Tocopherol) ist ein wichtiges fettlösliches Antioxidans. Eine präklinische antiproliferative Wirkung beim Prostatakarzinom ist lediglich durch eine gewisse antiandrogene Wirkung wahrscheinlich

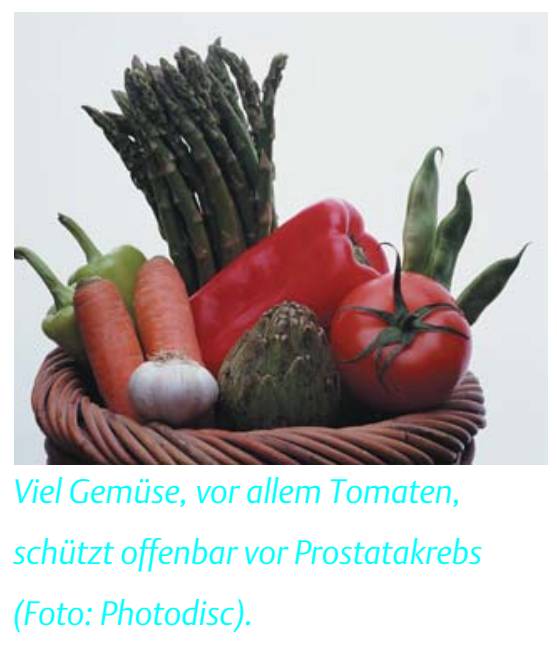

(Thompson TA, Wilding G. Mol Cancer Ther 2003; 2: 797-803). Große Studien zur Prävention von Lungenkrebs zeigten jedoch eine signifikante Reduktion der Inzidenz des Prostatakarzinoms in der Interventionsgruppe mit Vitamin E (um 32\%) sowie eine höhere Inzidenz bei Probanden mit niedrigen $\alpha$-Tocopherol-Spiegeln (Goodman GE et al. Cancer Epidemiol Biomarkers Prev 2003; 12: 518-526).
Selen als Spurenelement ist in vielen antioxidativ wirkenden Enzymen enthalten. Es wird durch Verzehr von Gemüse und Fleisch von Pflanzen fressenden Tieren aufgenommen. Der wesentliche Hinweis für die Bedeutung von Selen beim Prostatakarzinom kam aus einer Studie zur Krebsprävention bei Patienten mit nichtmelanomatösem Hautkrebs, in der in der Behandlungsgruppe mit oraler Selennahrungssupplementierung eine signifikante Reduktion der Prostatakarzinominzidenz festgestellt wurde (odds ratio 0,48) (Duffield-Lilico AJ et al. BJU Int 2003; 91: 608-612).

Zwei gegenwärtig laufende Studien zur Prävention des Prostatakarzinoms sind aufgrund dieser Hinweise auf die mögliche Bedeutung von Selen und Vitamin E aufgelegt worden. Die erste Studie (SWOG 9917), durchgeführt von der South Western Oncology Group (SWOG) in den USA, untersucht den möglichen Effekt einer täglichen Nahrungssupplementierung mit $200 \mathrm{mg}$ Selen bei Männern mit nachgewiesenen präkanzerösen Veränderungen („high grade PIN“) über einen Zeitraum von 4 Jahren bei 450 Probanden. Die zweite Studie wird vom amerikanischen National Cancer Institute gefördert und umfasst eine plazebokontrollierte Prüfung von Selen und Vitamin E allein oder in Kombination bei über 32000 Probanden mit einem Ausgangs-PSA-Wert < $4 \mathrm{ng} / \mathrm{ml}$; das Studienende liegt im Jahr 2014 (SELECT, Selenium and Vitamin E Cancer Prevention Trial)(Klein Eet al. WorldJUrol 2003; 21: 21-27).

\section{Chemoprävention mit endokrin wirksamen Medikamenten}

Aus der Hormonabhängigkeit des Prostatakarzinoms ergibt sich auch die potentielle Möglichkeit der Chemoprävention des Prostatakarzinoms durch endokrin wirksame 
Tab. 2 Prospektive Interventionsstudien zur Prävention des Prostatakarzinoms

\begin{tabular}{|c|c|c|c|c|}
\hline Studie & $\begin{array}{l}\text { Anzahl } \\
\text { Probanden }\end{array}$ & Einschlusskriterien & Intervention & Ergebnis \\
\hline PCPT Trial & 18882 & $\begin{array}{l}\text { PSA-Ausgangswert } \\
<3 \mathrm{ng} / \mathrm{ml}\end{array}$ & $\begin{array}{l}\text { Finasterid } 5 \mathrm{mg} / \mathrm{d} \\
\text { vs. Plazebo über } \\
7 \text { Jahre }\end{array}$ & $\begin{array}{l}\text { Reduktion der Prostatakarzi- } \\
\text { nomrate um insgesamt } 24,8 \% \text {, } \\
\text { höhere Rate an schlecht } \\
\text { differenzierten Karzinomen }\end{array}$ \\
\hline $\begin{array}{l}\text { SWOG } \\
9917\end{array}$ & 450 & $\begin{array}{l}\text { prostatische intraepitheliale } \\
\text { Neoplasie (high grade) }\end{array}$ & $\begin{array}{l}200 \mu \mathrm{g} \text { Seelen/d } \\
\text { vs. Plazebo }\end{array}$ & voraussichtlich 2007 \\
\hline PRP-1 & 306 & $\begin{array}{l}\text { prostatische intraepitheliale } \\
\text { Neoplasie (high grade), } \\
\text { PSA }<10 \mathrm{ng} / \mathrm{ml}\end{array}$ & $\begin{array}{l}200 \text { mg Selen } \\
\text { + Soja } 40 \text { mg } \\
\text { + Vitamin E } 800 \text { IE }\end{array}$ & voraussichtlich 2008 \\
\hline $\begin{array}{l}\text { SELECT } \\
\text { Trial }\end{array}$ & 32453 & $\begin{array}{l}\text { PSA-Ausgangswert } \\
<4 \mathrm{ng} / \mathrm{ml}\end{array}$ & $\begin{array}{l}\text { Selen und/oder Vitamin } \\
\text { E, plazebokontrolliert }\end{array}$ & voraussichtlich 2014 \\
\hline $\begin{array}{l}\text { REDUCE } \\
\text { Trial }\end{array}$ & 8000 & $\begin{array}{l}\text { PSA-Ausgangswert } \\
4-10 \mathrm{ng} / \mathrm{ml} \text {, } \\
\text { negative Biopsie }\end{array}$ & $\begin{array}{l}\text { Dutasterid 0,5 mg } \\
\text { vs. Plazebo über } \\
\text { 4 Jahre }\end{array}$ & voraussichtlich \\
\hline
\end{tabular}

en ein Prostatakarzinom zu entdecken, ist größer bei kleineren Organvolumina. Daraus resultiert ein möglicher systematischer Bias in dieser Untersuchung. Aufgrund der Ergebnisse dieser Studie wird jedoch eine präventive Einnahme von Finasterid derzeit weder von der Deutschen Gesellschaft für Urologie noch von der European Association of Urology empfohlen.

Mit der gleichen Hypothese wird in einer internationalen Studie der duale $5 \alpha$-Reductasehemmer Dutasterid untersucht. Dabei werden 8000 Probanden mit einem Ausgangs-PSA-Wert von 4-10 ng/ml und einer negativen Prostatabiopsie plazebokontrolliert über 4 Jahre behandelt, mit Kontrollbiopsien nach 2 und 4 Jahren(REDUCE Studie).

Eine antiproliferative Wirkung verspricht man sich in vielerlei Hinsicht auch von Substanzen, die in den Arachidonsäurestoffwechsel eingreifen. Die Cyclooxygenase-Typ 2 ist auf prostatischen intraepithelialen $\mathrm{Ne}$ oplasien(PIN) überexprimiert(Shappell SB et al. Cancer Res 2003; 63: 2256-2267). Eine groß angelegte, von der Industrie geförderte Studie zur Chemoprävention des Prostatakarzinoms mit dem COX2-Hemmer Rofecoxib wurde mittlerweile jedoch wegen der kardiovaskulären Toxizität der Substanz eingestellt, da die Probanden nicht entsprechend selektioniert waren.

Fazit

Zusammenfassend gibt es zunehmende Evidenz dafür, dass präventive Maßnahmen beim Prostatakarzinom möglich und erfolg- versprechend sind. Die gegenwärtig laufenden Studien werden hierzu weitere aufschlussreiche Daten liefern. Schon jetzt kann festgestellt werden, dass diätetische Maßnahmen mit Fleisch- und Fettreduktion zugunsten von Gemüse und Obst sowie Fisch empfehlenswert sind. Zusätzlich gibt es genügend klare Hinweise dafür, dass gekochte Tomaten (Lycopene) und besonders Sojamilch zur Prävention empfohlen werden können. Hinweise, dass auch Selen und Vitamin als Nahrunsgssupplemente günstig erscheinen, liegen ebenfalls vor, jedoch werden weitere Studien Klarheit bringen müssen. Inwieweit eine medikamentöse Prävention mit $5 \alpha$-Reductasehemmern in Zukunft neue Aspekte eröffnet, muss abgewartet werden.

Korrespondenz

Priv.-Doz. Dr. med. O. W. Hakenberg Klinik und Poliklinik für Urologie Universitätsklinikum Dresden Fetscherstr. 74, 01307 Dresden DOI: $10.1055 / \mathrm{s}-2005-872618$

Fachliche Betreuung der
„Serie Prävention“保
Prof. Dr. Dr. h. c. Peter C. Scriba
Medizinische Klinik / Klinikum Innenstadt
der Universität München
Ziemssenstr. 1
80336 München
Prof. Dr. Friedrich W. Schwartz
Medizinische Hochschule Hannover
Abteilung für Epidemiologie, Sozialmedizin
und Gesundheitsforschung
Carl-Neuberg-Str. 1
30623 Hannover

